Forum 2016 · 31:345

DOI 10.1007/s12312-016-0101-1

Online publiziert: 18. Juli 2016

๑) Springer-Verlag Berlin Heidelberg 2016

KREBSVERBAND

: BADEN-WÜRTTEMBERG E.V.

Krebsverband Baden-Württemberg e.V., Stuttgart

\title{
Reha-Netz JuKiE - Eine Chance für Schule, Studium oder Beruf
}

zielt. Acht von zehn betroffenen Kindern und Jugendlichen werden inzwischen geheilt. Eine erfolgreiche Krebstherapie und lange Behandlungszeiten können aber in der Folge gravierende körperliche und seelische Folge- bzw. Bewältigungsprobleme hinterlassen. Ohne qualifizierte Nachbetreuung und Unterstützung drohen die Erfolge der Akutmedizin nur Stückwerk zu bleiben. Doch man muss diese besonders belasteten jungen Menschen erkennen, deren Probleme sorgfältig erfassen, ihre Leistungsfähigkeit testen und unter realitätsnahen Bedingungen erproben. Wenn nötig auch länger als die üblichen 4 Wochen. Gerade bei den jungen Erwachsenen sollten Aussichten auf eine Wiedereingliederung ins Berufsleben ermöglicht werden.

\section{Neue Wege der Kooperation}

Die am Netzwerk beteiligten Rehabilitationskliniken haben sich sehr konsequent auf die Therapie dieser schwer- und chronischkranken Patienten und ihrer Familien spezialisiert. Im Verbund dieser Einrichtungen konnten neue Wege der Kooperation in der Rehabilitation beschritten werden:

Während des Rehabilitationsaufenthaltes der Kinder und Jugendlichen in der Klinik Katharinenhöhe, der Rehaklinik am Kurpark oder der Nachsorgeklinik Tannheim wird der mögliche Bedarf an einer schulischen/beruflichen Neuorientierung durch ein systematisches Screening erkannt. Den Rehabilitanden wird angeboten an einer schulisch/beruflichen Orientierungsphase in Verbindung mit bzw. im Anschluss an die ,klassische“ Rehabili- tationsmaßnahme im Hegau-Jugendwerk teilzunehmen. Diese Orientierungsphase wird unter Einsatz von modernen Assessment-, Testungs- und Übungsverfahren durchgeführt.

Dabei wird ganz individuell ein Überleitungsprogramm sowie ein Wiedereingliederungsplan erstellt durch die Rehaklinik, in Kooperation mit dem Hegau-Jugendwerk und/oder mit weiteren Partnern wie der Arbeitsagentur, Integrationsfachdiensten, Beratungsstellen, Akutkliniken, Berufsbildungs- und Berufsförderungswerken.

Der Aufenthalt in der Katharinenhöhe/Nachsorgeklinik Tannheim (Kinder und Jugendliche) ist in der Regel vierwöchig, in der Rehaklinik am Kurpark (junge Erwachsene) in der Regel dreiwöchig. Für Versicherte der Deutschen Rentenversicherung Baden-Württemberg auch verlängerbar.

Für eine gezielte Auswahl an Rehabilitanden folgte eine schulisch/beruflichen Orientierungsphase im Hegau-Jugendwerk.

Auch wenn JuKiE als eigenständiges Projekt Ende 2013 beendet wurde, bestehen die Kooperationen weiter, werden intensiviert und hat über Baden-Württemberg hinaus zahlreiche Impulse gegeben. Für die DRV Baden-Württemberg wurde es in die Regelversorgung übernommen.

\section{Kontakt}

Krebsverband Baden-Württemberg e.V. Adalbert-Stifter-Straße 105, 70437 Stuttgart Tel: 0711 848-10770

Fax: 0711 848-10779

E-Mail: info@krebsverband-bw.de

Internet: www.krebsverband-bw.de 